\title{
Téoros
}

Revue de recherche en tourisme

\section{D'un concept de parc thématique sur la cité du Père Noël à un parc sur la nordi-cité}

\author{
Suzanne Chassé, le Groupe Promexpro inc., le Comité de la cité internationale \\ du Père Noël, le ministère du Tourisme du Québec et la Ville de Laval
}

Volume 12, numéro 3, octobre 1993

Le renouveau des parcs à thèmes

URI : https://id.erudit.org/iderudit/1077936ar

DOI : https://doi.org/10.7202/1077936ar

Aller au sommaire du numéro

Éditeur(s)

Université du Québec à Montréal

ISSN

0712-8657 (imprimé)

1923-2705 (numérique)

Découvrir la revue

Citer cet article

Chassé, S., le Groupe Promexpro inc., le Comité de la cité internationale du Père Noël, le ministère du Tourisme du Québec \& la Ville de Laval (1993). D’un concept de parc thématique sur la cité du Père Noël à un parc sur la nordi-cité. Téoros, 12(3), 32-36. https://doi.org/10.7202/1077936ar 


\section{D'un concept de parc thématique sur la cité du Père Noël à un parc sur la nordi-cité}

Suzanne Chassé"

en collaboration avec le Groupe Promexpro inc., le Comité de la cité internationale du Père Noël, le ministère du Tourisme du Québec et la Ville de Laval"

\section{Introduction}

Promexpro est l'une des plus importantes entreprises québécoises spécialisées dans la création et l'organisation d'expositions et d'événements spéciaux au Québec.

Depuis sa fondation en 1979 , avec la réalisation du premier Salon national de l'habitation, l'entreprise est passée maitre dans l'art de créer, concevoir et réaliser des expositions.

En 1987, Promexpros'intéresse au marché du divertissementavecla préoccupation de créer à Montréal des événements pour la famille.

Suite à la réalisation de trois grands événements, monsieur Pierre Parent, président et chef de la direction, conçoit le projet d'un parc thématique relié aux thèmes du Père Noël et d'un pays nordique avec l'objectif de doter le Québecd'un attrait touristique à grand pouvoir de rétention.

Après plusieurs mois de développement et de recherche sur les pares thématiques, le projet préliminaire est présenté à différentes personnalités politiques, hommes d'affaires et professionnels qui, en conclusion, manifestent de l'intérêt à voir le projet se développer et à y collaborer.

Un comité de conceptualisation composé despécialistesen divertissement, marketing, architecture et concepteurs est formé afin d'élaborer le projet.

\section{La Cité internationale du Père Noël}

Un jour, nous avons tous cru au Père Noël en imaginant son village, d'y rencontrer ses lutins, son renne, Mère Noèl ou encore de le voir passer au-dessus de notre maison. Enfin, pourquoi ne pas admettre que nous

Madame Suzanne Chassd est protesseure en tourisme au College de Grantry et assocife-expert chez Zins, Beauchesne et associés.

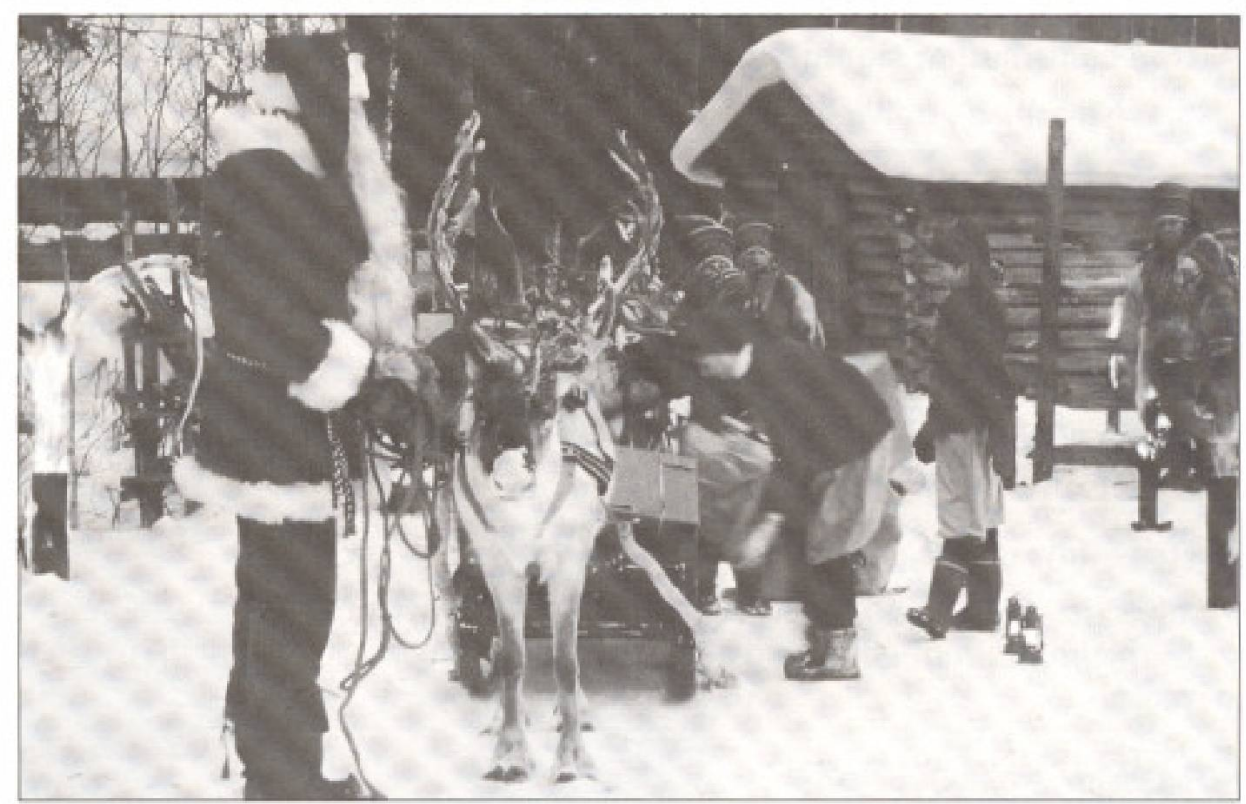

aimerions, ne serait-ce qu'un instant, qu'il existe vraiment!

Après tout, lorsqu'arrive la magie du temps des fêtes, ne travaillons-nous pas très fort pour recevoir cet invité de marque?

Le Père Noèl est un personnage neutre, pacifique et immortel qui provoque l'imagination et la fantaisie. Mais où vit le Père Noell?

Pourquoi pas au Québec... puisque c'est au Québec, en 1977, que Poste Canada met sur pied un programme de lettres-réponses traduites dans toutes les langues pour répondre au courrier venant de partout à travers le monde et qui est adressé au Père Noél, Pôle Nord, HOH OHO.

Le Comité s'est donc interrogé sur la faisabilité de créer un attrait majeur basé sur une cité internationale du Père Noèlen développant sept pôles thérmatiques:

- un pôle du Père Noel, au coeur du parc;

un pôle nordique, axé sur la nature et la culture des zones nordiques; un pôle des sciences, axé sur les saisons, la météo, les latitudes nordiques;

un pôle de jeux et jouets;

un pôle du sport;

un pôle des villes nordiques;

un pôle de la paix.

Le défi était de concevoir un site qui serait en opération douze mois par année et où la neige et le froid seraient des éléments positifís et valorisés de façon à accroîtte non seulement le tourisme national mais le tourisme international.

Un an plus tard, afin de valider le concept, une étude de préfaisabilité a étế réalisée et elle devait apporter des réponses à plusieurs interrogations afin de :

- définir plus spécifiquement le concept de parc thématique centré sur le du Père Noël, la nordicité et les enfants;

- déterminer le potentiel de marché et les marchés cibles du parc;

- analyser les attraits concurrentiels;

- déduire les critères de performancés d'un produit à succès au Québec; 
- déterminer le niveau optimal d'activité du parc et les critères de performance spécifiques qui y sont reliés en termes de taille, services offerts, accessibilité, etc.;

- définir les limites de la thématique et des produits et services offerts;

- définir les paramètres de calibrage du projet en matière d'investissement;

- élaborer sommairement les scénarios de rentabilité et vérifier la viabilité financière du projet;

- évaluer le potentiel du parc comme tremplin pour le tourisme au Québec et dans sa région d'implantation.

Pour y parvenir, plusieurs entrevues et analyses ont été réalisées auprès de différents groupes et une recherche de données a été complétée afin de:

- faire un inventaire des attraits comparables et concurrents en Amérique du Nord et en Europe, et de procéder à une analyse de leurs caractéristiques, leurs stratégies et leur performance,

- recueillir les perceptions des intervenants touristiques du Québec;

- recueillir les perceptions et l'accueil réservéau projetde Citédu Père Noël par les tours-opérateurs (français, américains, etc.) desservant les touristes venant au Québec;

- identifier les tendances des parcs thématiques et des attraits concurrentiels quant au thème, aux superficies, aux critères de succès, à la gestion, etc.;

- faire un portrait de l'industrie des jouets en identifiant les catégories de jouets que l'on y trouve, les foires commerciales et les tendances de ce marché:

- caractériser les périodes d'ouverture en fonction des sites comparables, des marchés et de la saisonnalitế;

- énumérer des critères d'implantation qui devront être pris en compte dans le choix d'un site.

\section{Constats sur la faisabilité du concept initial}

\section{En fonction des différents pôles}

Les résultats des analyses de chacun des pôles se fondent sur une évaluation des prérequis de chaque pôle, d'une analyse de parcs et attraits concurrents, d'entrevues avec des intervenants touristiques régio- naux et d'entrevues avec des tours-opérateurs internationaux.

Le pôle du Père Noèl est un pôle dont l'attraits'exercerait plutôtsur les familles et les enfants tout au cours de l'année et sur l'ensembledela population durant la courte période des fêtes. Le thème du Père Noël ne s'est pas révélé un thème porteur car c'est une thématique qui a été modifiée ou abandonnée dans plusieurs parcs américains l'ayant déjà exploitée.

Par contre, il existe un village du Père Noël en Laponie (Rovaniemi) et le grossiste Scanditour intègre cepersonnagedans son forfait. Le Père Noël n'y est pas l'unique attrait mais plutôt une *mascotte» ou un *emblème»; l'attrait réel est, en saison estivale, le soleil de minuit et la route vers le Cap Nord, et l'hiver, la pratique d'activités comme la motoneige, le ski de fond et le traîneau tiré par les chiens.

\section{Le pôle nordique}

Ce pôle est celui qui présente le plus d'intérêt auprès desclientèles et pourrait être le plus distinctif pour le Québec avec des points d'intérêt particuliers tels que le zoo d'animaux nordiques, l'aquarium nordique et la mise en valeur des us ct coutumes des autochtones.

Ce thème a l'avantage de pouvoir rejoindre un vaste marché de clientèles québécoises et internationales, jeunes et adultes, mais à la condition de respecter «l'authenticités du sujet.

\section{Le pôle des sciences}

Le pôle des sciences peut, tout comme le pôle nordique, présenter un intérêt certain pour une grande variété de clientèles, à condition de:

se distinguer de ses concurrents en mettant en valeur des thếmatiques et phénomènes nordiques, et tout particulièrement arctiques;

- faire une présentation vivante mais irréprochable des thèmesscientifiques abordés.

\section{Le pôle des jeux et des jouets}

Ce pôle bien traité, de façon historique, culturelle, technique et esthétique, peut s'adresser autant à une clientèle d'adultes que de jeunes. Des éléments d'intếrêt pourraient être:

- un musée du jouet;

. un pavillon des plus beaux jouets du monde;

- des manèges pour enfants et adolescents.

Par contre l'organisation actuelle, et bien établie, de l'industrie du jouet au niveau international ne permet pas d'entrevoir de grandes chances de succés dans l'établissement d'une foire permanente sur le site, pour les acheteurs du monde entier ou encoreen créant une ville d'usines de jouets.

\section{Le pôle des sports}

Ce pôle a l'inconvénient d'être difficile à intégrer dans un concept de parc car le Québec offre des possibilités de pratique réelle de la motoneige, de ski, et de traîneau. Les clientèles internationales sont d'ailleurs très attirées par cette pratique même si elle est offerte sous forme d'initiation. Une concurrence très forte existe déjà au niveau des activités sportives et de plein air d'été au Québec.

\section{Le pôle des villes nordiques}

Ce pôle s'adresse davantage à une clientèle adulte; une approche plus typique et représentant davantage des villages offrirait un intérêt plus grand auprès de la dientèle.

\section{Le pôle de la paix}

Ce pôle est le plus controversé des sept pôles, d'une partà cause des difficultés liées à sa mise en oeuvre et d'autre part, parce que son impact touristique n'est pas très fort.

\section{En fonction du marché potentiel}

En termes de potentiel relatif de marché et de taux de pénétration prévisible, on peut classer les pôles en deux grandes catégories:

\section{Fort potentiel}

- Pôle Nordique et Arctique;

- Pôle des Jeux et des Jouets;

- Pôle des Villages Nordiques;

- Pôle des Sciences. 


\section{Potentiel modéré}

- Pôle du Père Noèl;

- Pôle des Sports;

- Pôle de la Paix.

Bien entendu, le classement de ces catégoriestientcompted'estimations brutes, d'un effort raisonnable de mise en marché et d'un concept à valeur élevée. Un caractère particulier donné à chaque pôle pourrait insuffler plus de force au pôle; par contre, chacun de ces pồles traité sans originalité peut s'avérer devenir un attrait nul.

\section{Constats et diagnostics}

\section{CONSTATS}

Un parc thématique intégrant l'ensemble des éléments thématiques proposés dans un seul lieu bien défini, peut présenter un certain nombre d'inconvénients:

d'abord, pour bien traiter chacun des pôles et en assurer une bonne intégration et un bon positionnement concurrentiel, on se retrouve devant un investissement colossal pour le Québec;

ensuite, l'authenticité requise pour plusieurs des pôles risque de souffrir si l'ensemble de la thématique doit être traité dans un espace restreint, surtout si l'on considère qu'au Québec la nordicité existe réellement et qu'elle peut être observée et vécue. Il en est de même pour les activités sportives qui peuvent être pratiquées dans nos grands espaces.

\section{Le positionnement global}

Le positionnement global du parc envisagé devra au départ être très concentré et à fort impact et ne pas être le résultat d'un assemblage fortuit de diverses thématiques. Un exercice important d'articulation des thématiques doit être conduit afin que le visiteur sache ce qu'il vient voir, et comprenne ensuite le sens de sa visite dans le parc. Il doit aussi pouvoir distinguer ce qui est artificiel de ce qui est didactique, et de ce qui est tout simplement là pour son plaisir.

La force d'un tel positionnement est une des principales garanties de succès d'un projet comme celui envisagé par les promoteurs.

Tableau 1

Potentiel relatif des différents pôles

\begin{tabular}{|c|c|c|}
\hline Pôles & $\begin{array}{c}\text { Marchés } \\
\text { potentiels } \\
\text { (en millions) }\end{array}$ & $\begin{array}{l}\text { Force de } \\
\text { pénétration } \\
\text { potentielle }\end{array}$ \\
\hline
\end{tabular}

Pöle nordique et arctique

Pôle des jeux et jouets

Pồle des villes nordiques

Pôle des sciences

Pôle du Père Noël

Pôle des sports

Pôle de la paix

79,0
79,0
79,0
65,0
8,1
6,4
11,6

79,0

79,0

79,0

65,0

6,4

11,6
Fort
Fort
Modéré
Modéré
Faible
Modéré
Faible
Excellente
Bonne
Moyenne
Moyenne
Moyenne
Faible
Difficile

\section{Les marchếs et dientèles}

Le marché potentiel du parc thématique prévu est très important, pour toutes les clientèles visées, autant locales que touristiques. La variété de ces clientè̀les en termes de profils peut représenter une assurance supplémentaire de succès.

Toutefois, la couverture optimale de ces marchés est déjà atteinte par les 3 ou 4 premiers pôles en termes d'attrait: pôle nordique, pồle des sciences, pồle des villes nordiques et pôle des jouets.

Les autres pôles ne viennent qu'ajouter une force d'attraction marginale: sports, Père Noël et paix. Et pour les sports surtout, si l'on reste à l'intérieur d'un parc thématique, l'attrait restera sous-optimisé.

\section{La localisation géographique}

La localisation pose également un problème particulier car pour préserver l'authenticité, une partie des installations devrait être en plein air et, pour des raisons climatiques, la localisation devrait alors se déplacer vers des territoires plus au nord de Montréal perdant ainsi de son accessibilité et de son pouvoir d'attraction auprès du marché touristique et des résidants de Montréal.

\section{DIAGNOSTICS}

\section{Forces et atouts du projet}

originalité d'une série de thématiques très actuelles, en particulier l'aspect de la nordicitế; correspondance dela thếmatiqueavec la réalité du Québec;

possibilité de développer un véritable avantage concurrentiel;

- importance du marché potentiel.

\section{Faiblesses et contraintes}

- importance des investissements requis et financement;

- défi de la promotion d'un nouvel attrait de cette nature sur le marché compte tenu des budgets et de la concurrence;

- difficulté de répondre dans un espace restreint, auxexigencesd'authenticité requises par les marchés;

- problématique de la localisation qui doit trouver un site optimisant les facteurs: espace, climat, accessibilité, proximité du marché.

\section{Opportunités}

- intérêt croissant pour la nature et les cultures nordiques prowenant de la clientèle internationale;

- absence d'un attrait mettanten valeur la nordicité québécoise.

\section{Menaces}

- intérêt mitigé de la part du marché québécois pour l'hiver;

déclin de l'attrait global des parcs thématiques (diminution du taux de croissance);

- faiblesse d'attraction de la destination Québec/Montréal dans un contexte international; 
conditions climatiques;

évolution culturelle des enfants pour le personnage du Père Noël.

\section{Enjeux et défis}

trouver une thématique intégrée préservant la forced'attrait, l'authenticité et l'originalité, afin de développer un attraitayantun avantage concurrentiel majeur sur un marché international; trouver une façon d'intégrer l'authenticité et la concentration géographique;

maintenir les investissements à un niveau permettant leur financement et une exploitation non déficitaire.

\section{Réorientation du projet}

Suite au diagnostic posé et aux enjeux qui en découlent, des recommandations ont été formulếes pour la poursuite du projet:

- Le projet pourrait être poursuivi à condition d'effectuer une adaptation majeure en termesdepositionnement de la thếmatique centrale et de l'établissement géographique.

- Le positionnement devrait êture développé autour de la nordicité: son expression dans la natureet la culture, sa célébration par Noël, ainsi que sa symbolique (espace, paix, harmonie, écologie, etc.).

- Les thếmatiques devraient être intégrées dans un continuum:

- la nordicité : sa compréhension scientifique, sa révélation $\mathrm{d}$ a $\mathrm{ns}$ la nature, ainsi que son impact et son expression dans la culture, les modes de vies, les activités et l'architecture;

- le solstice d'hiver et ses célébrations (Noël, légendes, Père Noël, etc.) ainsi que les différents aspects de cette célébration (arbres de Noël, jouets, décorations, crèches, santons, etc.);

les sports nordiques: le nord comme plaisir;

les enfants et leurs droits, ceux qui croient le plus au $\&$ Père Noëls, qui osent rêver à la paix, à l'environnement, à l'éducation et à l'harmonie.

- Un concept éclaté, qui permettrait de combinerà l'intérieur dela thématique proposée, un ensemble d'attraits et d'activités qui ne soient plus contraints géographiquement. Ceconceptéclaté permettrait de combiner authenticité et approche didactique, par un centre intégrateur à valeur didactique et des centres éclatés à teneur expérimentale.

\section{Nouveau concept}

- Àla suite derésultats, des commentaires obtenus et des discussions du Comitế, un nouveau concept a été développé. Le thème du Père Noẻl ne serait donc pl us retenu comme thème majeur du parc, il deviendrait acoessoire au thème principal. Le thème retenu serait celui de la nordicité et l'appellation pourrait être la suivante: le Centre de la NORDI-CITE.

- Le concept ne se restreindra plus exclusivement à un parc thématique traditionnel, mais sera complété par des activités annexes. Le Centre de la NORDI-CITÉ sera développé en fonction de trois concepts:

un site intégrateur, soit le parc de loisirs;

un tremplin pour le positionnement du Québeç

des sites satellites liés dans un réseau (attraits situés dans les différentes régions du Québec).

\section{Site intégrateur}

Le siteintégrateur serait un parcthématique qui nécessiterait l'immobilisation de sommes importantes, le thème qui prédominerait serait relié à la nordicité.

Autour de cette thématique, d'autres pôles viendront s'ajouter pour alimenter le sujet principal. Différentes approches devront être envisagées pour l'exploitation de ces pôles: approches culturelle, scientifique, ludique, imaginaire.

\section{Approcbe culturelle}

- pôle privilégiant la connaissance des territoires nordiques et arctiques;

- pavillons sur les habitudes de vie des Inuit et des Cris;

- films sur la nordicité (artisanat, igloo, baleinière, trappe, etc.).

\section{Approche scientifique}

planétarium et phénomènes visibles au-dessus du $55^{\circ}$ parallèle; métóorologie et conditions climatiques des sommetset des latitudesnordiques et arctiques.

\section{Approcbe ludique}

villages nordiques; prétexteà l'animation, aux achats, à la restauration (boutiques, spectacles, etc.).

\section{Apprache inaginaire}

l'hiver est une saison associće à la fête de Noël et à son personnage fantastique (Père Noël, Saint-Nicolas, SantaClaus, etc.). Sous cette approche, on exploitera ainsi l'arbre de Nö̈l et ses décorations, les crèches dans les pays chrétiens et le monde amusant des jouets (salle de montre, pavillon d'animation, etc.).

\section{Tremplin}

À partir de la thématique du Centre de la NORDI-CITÉ, toute une série d'activités complémentaires pourront être offertes. A l'origine, le pôle des sports devait offrir un éventail d'activités sportives et de plein air.

Il faudrait prévoir soit la création d'une compagnie ou entente avec un grossiste déjà existant afin de créer des forfaits à partir d'activités connexes au thème de la nordicité (activités de plein air, culturelles, etc.).

À titre d'exemplevoici une série de forfaits possibles :

- camping d'hiver dans un igloo;

- descente de rivières en kayak;

- séjour de trappechez lesAmérindiens;

- randonnée pédestre;

- circuit demotoneige dans le réseau de sentiers le plus important au monde;

- croisières nordiques, etc.

Ce grossiste offrirait aux clientèles intéressées, un séjour lié à la pratique d'activités nordiques. Il permetrrait d'intégrer la thématique du parc thématique, dans le produit touristique québéoois et permettrait aux régions de bénéficier de la promotion duparcthématique. Lesiteintégrateur servirait de levier pour promouvoir des vacances au Québec. 


\section{Sites satellites}

En plus de la visite du parc thématique, des ententes pourraient être conclues avec des responsables d'attraits liés à la nordicité, dans le but de créer un réseau reconnu à travers tout le Québec.

Un regroupement ou une bannière pourrait être créé permettant ainsi de mieux orienter les visiteurs et de promouvoir plus efficacement chacune des composantes. Une stratégie de service serait commune à tous ces sites (accueil, etc.).

Sous la bannière du Centre de la NORDICITÉ, plusieurs attraits déjà existants pourraient adhérer à ce regroupement tels que les villages et musées amérindiens (Pointe Bleue, Odanak, etc.), le village d'antan de Drummondville, les pourvoiries, le village de pêche blanche, les cabanes à sucre, le jardin zoologique, les boutiques d'artisanat et d'art Inuit, etc.

\section{Conclusion}

En conclusion des premiers rapports de la firme, le Comité constate que la population préfere recevoir la visite du Père Noël que de se déplacer pour le rencontrer.

Malgré les forces et l'originalitê du projet, la prudence est recommandée étant donné le déclin de l'attrait pour les parcs thématiques et la faiblesse d'attraction de la destination Québec/Montréal dans un contexte international.

Le Comité a retravaillé le projet selon les recommandations en proposant un concept éclaté avec un parc intégrateur et des sites et activités satellites choisis à travers le Québec.

Le projet devient la nordi-cité où le Père Noël devient un sous-thème. Cette nouvelle vision s'adresse davantage à la compétence des tours opérateurs et Promexpro préfere continuer de réaliser des produits selon son expertise et sa mission.

Dans le contexte économique actuel et à venir, le succès du développement d'une entrepriserepose sur la qualité etla profondeur qu'elle alloueà une étude de faisabilité. Il ne faut pas craindre de pousser les nouvelles idées mais le danger est surtout d'engager trop vite des sommes importantes pour les réaliser.
Quelque soit les conclusions d'un rapport de faisabilité, si le projet ne peut pas être développé dans l'immédiat... c'est comme le Père Noël, il reviendra l'an prochain ou une autre année! 